\title{
Heuristics in Permutation GOMEA for Solving the Permutation Flowshop Scheduling Problem
}

\author{
G. H. Aalvanger ${ }^{1}$, N. H. Luong ${ }^{2}$, P. A. N. Bosman ${ }^{2}$, and D. Thierens ${ }^{1(\bowtie)}$ \\ 1 Institute of Information and Computing Sciences, \\ Universiteit Utrecht, Utrecht, The Netherlands \\ d.thierens@uu.nl \\ 2 Centre for Mathematics and Computer Science (CWI), \\ Amsterdam, The Netherlands
}

\begin{abstract}
The recently introduced permutation Gene-pool Optimal Mixing Evolutionary Algorithm (GOMEA) has shown to be an effective Model Based Evolutionary Algorithm (MBEA) for permutation problems. So far, permutation GOMEA has only been used in the context of Black-Box Optimization (BBO). This paper first shows that permutation GOMEA can be improved by incorporating a constructive heuristic to seed the initial population. Secondly, the paper shows that hybridizing with job swapping neighborhood search does not lead to consistent improvement. The seeded permutation GOMEA is compared to a state-of-the-art algorithm (VNS4) for solving the Permutation Flowshop Scheduling Problem (PFSP). Both unstructured and structured instances are used in the benchmarks. The results show that permutation GOMEA often outperforms the VNS4 algorithm for the PFSP with the total flowtime criterion.
\end{abstract}

\section{Introduction}

Recently, Bosman et al. [2] introduced the permutation Gene-pool Optimal Mixing Evolutionary Algorithm (GOMEA), a model-based evolutionary algorithm which is able to solve permutation problems from a Black-Box Optimization (BBO) perspective. Permutation GOMEA has been tested on the Permutation Flowshop Scheduling Problem (PFSP) with the total flowtime (TFT) criterion. In these tests, permutation GOMEA outperformed GM-EDA [3] another permutation model-based evolutionary algorithm. In order to improve permutation GOMEA further, we should shift from a BBO perspective to a White-Box perspective. In this paper we study the effect of seeding the initial population with solutions from a constructive heuristic, and we look at hybridizing permutation GOMEA with neighborhood search heuristics.

Section 2 briefly introduces permutation GOMEA. After this we explain the PFSP and benchmark instances and performance measures in Sect. 3. Constructive heuristics for the PFSP are given in Sect.4.1, along with some experiments

(C) Springer Nature Switzerland AG 2018

A. Auger et al. (Eds.): PPSN 2018, LNCS 11101, pp. 146-157, 2018.

https://doi.org/10.1007/978-3-319-99253-2_12 
on the effectiveness of these heuristics. In Sect. 4.3 we do the same for improvement heuristics for the PFSP. Finally, we compare the permutation GOMEA seeded with a constructive heuristic - with VNS4, a state-of-the-art algorithm for solving the PFSP in Sect. 5. Section 6 concludes this paper.

\section{Permutation GOMEA}

\subsection{Solution and Model Encoding}

Permutation GOMEA encodes solutions using a random-key encoding [2]. A permutation of $n$ variables is encoded as $r=\left(r_{1}, \cdots r_{n}\right)$, where each random key $r_{i} \in[0,1]$. The position of variable $i$ in the permutation is equal to the position of $r_{i}$ when $r$ is sorted in ascending order. Multiple random key encodings can encode the same permutation. For example, $r_{1}=(0.34,0.56,0.21)$ and $r_{2}=$ $(0.72,0.93,0.12)$ both encode $x=(3,1,2)$.

\subsection{Model Building}

The model used in permutation GOMEA is a linkage tree that models dependencies between problem variables in a hierarchical manner [9]. The root of the linkage tree is a set with all variables. Each node is recursively split up, ending in leaves containing only a single variable. Variables grouped in a node are assumed to be dependent, so optimal mixing can improve solutions effectively.

In permutation GOMEA, the linkage tree is built in each generation anew, by merging nodes starting at the bottom of the tree. The two sets $i$ and $j$ are merged which have the strongest dependency $\delta(I, J)$. For two variables $i$ and $j$, the dependency is composed of two factors: $\delta(i, j)=\delta_{1}(i, j) \cdot \delta_{2}(i, j)$. The first dependency factor is based on relative-ordering information in the population and is calculated using the entropy of the probability that variable $i$ is before variable $j$ in the population:

$$
\delta_{1}(i, j)=1-\operatorname{Entropy}\left(p_{i, j}\right) .
$$

The second dependency factor uses the average squared distance in random key values of variable $i$ and $j$ :

$$
\delta_{2}(i, j)=1-\frac{1}{n} \sum_{k=0}^{n-1}\left(r_{i}^{k}-r_{j}^{k}\right)^{2} .
$$

This results in a symmetric dependency measure between two variables, where high values indicate a high dependency. We can extend the dependency measure to calculate the dependency between two sets, by taking the average pairwise dependency of the variables in the sets:

$$
\delta(I, J)=\frac{1}{|I| \cdot|J|} \sum_{i \in I} \sum_{j \in J} \delta(i, j) .
$$




\subsection{Optimal Mixing}

To generate new solutions, permutation GOMEA uses Gene-pool Optimal Mixing (GOM) [9]. For each solution, permutation GOMEA takes every set in the linkage tree as a crossover mask. The values of the masked variables are then substituted by values from a random donor solution. For example, solution $r_{1}=(0.2,0.3,0.6,0.5)$ is changed using crossover mask $\left(x_{1}, x_{2}, x_{4}\right)$ and donor $r_{2}=(0.9,0.5,0.1,0.7)$ to $r_{1}^{\prime}=(\mathbf{0 . 9}, \mathbf{0 . 5}, 0.6, \mathbf{0 . 7})$. If such a change is not strictly improving a solution, the substitution is reverted. Thanks to the random keys encoding, optimal mixing always results in a feasible permutation.

If a solution is not improved using any crossover mask, permutation GOMEA will 'force' improvements using the best known solution so far. In this Forced Improvement (FI) phase, permutation GOMEA repeats optimal mixing but the best known solution is used as donor, instead of a random one. In order to improve convergence changes are accepted when they do not decrease the quality of the solution. FI is also entered if the best overall solution has not changed for $10+10 \cdot \log n$ generations (denoted with variable NIS = true in Algorithm 1 ).

With a probability of 0.1 , permutation GOMEA will 'scale' the random keys before substitution. Here, the values to substitute are scaled to a new interval. For example, scaling random keys $(0.9,0.5,0.7)$ to the interval $[0.3,0.5]$ results in $(0.5,0.3,0.4)$. Scaling allows permutation GOMEA to move a group of variables closer together in the permutation. Also, the random key diversity is improved in the population. Random key diversity is also ensured by re-encoding. After the GOM phase of permutation GOMEA, each random key gets a new value, while retaining the order of the random keys.

\subsection{Population Sizing Scheme}

When implemented, permutation GOMEA would look like the pseudocode in Algorithm 1. However, one needs to specify the population size before running the algorithm. Therefore, permutation GOMEA incorporates an exponential population sizing scheme [2]. In this scheme, a population is started with size $n_{\text {base }}$. Every four times this population is evaluated, a population with size $2 \cdot n_{\text {base }}$ is evaluated once. This pattern recurses, so population $i$ is evaluated four times as often as population $i+1$. Using such a scheme, no population size has to be estimated. When a population is converged, no evaluations are performed anymore for that population, allowing permutation GOMEA to evaluate more in the other populations.

\section{Permutation Flowshop Scheduling Benchmark}

The PFSP is concerned with finding the optimal solution for scheduling $J$ jobs on $M$ machines. Each job requires $M$ operations, which should be performed sequentially, starting on machine 1 and finishing on machine $M$ (the Flowshop property). Operations cannot be interrupted, but a job can be delayed when its 


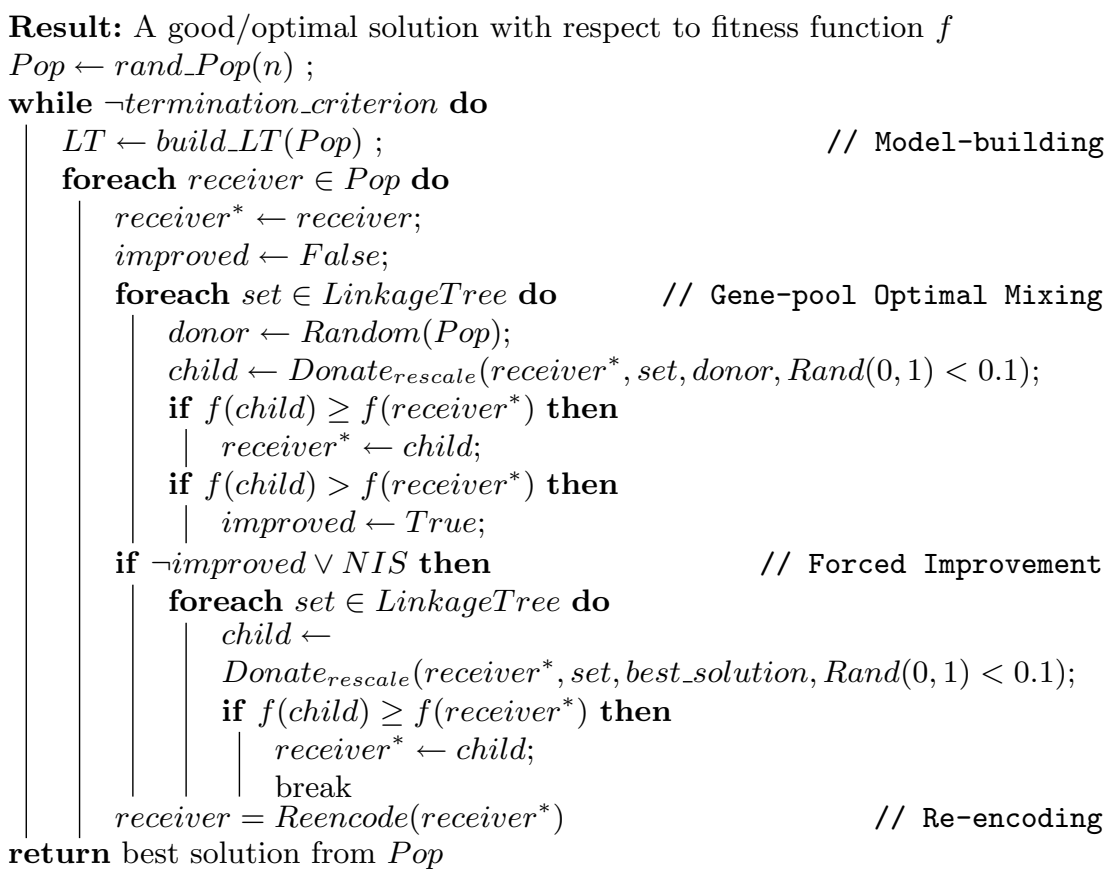

Algorithm 1. GOMEA outline

operations are not performed immediately after each other. Any solution can be seen as a permutation of jobs, since each machine has to process the jobs in the same order (the Permutation property). In three field notation, the PFSP is denoted by $F \mid$ prmu $\mid \gamma$, where $\gamma$ refers to the objective function that is used for optimizing the schedule. Here, we consider the total flowtime (TFT) criterion, which is defined as the sum of completion times of all jobs:

$$
T F T(\pi)=\sum_{i=1}^{J} c\left(\pi_{i}, M\right)
$$

The completion times of all jobs can be calculated using the equations in (5) in $\mathcal{O}(J \cdot M)$ time. For the TFT criterion, the PFSP is NP-hard when $M>1$.

$$
\begin{aligned}
& c\left(\pi_{1}, 1\right)=p\left(\pi_{1}, 1\right) \\
& c\left(\pi_{1}, j\right)=c\left(\pi_{1}, j-1\right)+p\left(\pi_{1}, j\right) \quad \text { for } j=2 \cdots M \\
& c\left(\pi_{i}, 1\right)=c\left(\pi_{i-1}, 1\right)+p\left(\pi_{i}, 1\right) \quad \text { for } i=2 \cdots J \\
& c\left(\pi_{1}, 1\right)=\max \left\{c\left(\pi_{i-1}, j\right), c\left(\pi_{i}, j-1\right)\right\}+p(\pi, j), \\
& \quad \text { for } i=2 \cdots J ; \text { for } j=2 \cdots M .
\end{aligned}
$$

Here, $p\left(\pi_{i}, j\right)$ is the processing time of job $\pi_{i}$ on machine $j$. The completion time of job $\pi_{i}$ on machine $j$ (i.e., $c\left(\pi_{i}, j\right)$ ) is the duration from when job $\pi_{i}$ is started on the first machine until job $\pi_{i}$ is finished on machine $j$. 


\subsection{Problem Instances}

\section{Taillard Instances}

For the PFSP, the most often used benchmark set is developed by Taillard [7]. This benchmark set can be divided in $12(J \times M)$ sets with 10 instances each (See Table 1). The instances are a selection of the hardest randomly generated instances. Here, instances for which simple metaheuristics do not often find the same solution or where the solution is far from a lower bound are considered to be hard.

\section{Structured Instances}

Aalvanger [1] introduced a new set of benchmarks for testing algorithms on structured instances. The benchmark set contains the three types of structured instances as described by Watson [10]: Job-correlated (JC), Machinecorrelated (MC) and Mixed-correlated (MXC) instances (see Fig. 1). In jobcorrelated instances, processing times are dependent on the job and not on the machines. Therefore the processing times of operations in one job are related. In machine-correlated instances the structure goes the other way around. Here, processing times on one machine are related, while processing times within one job are unrelated. Mixed-correlated instances are equal to Machine-correlated instances, but here the relative ranks of processing times within each machine are job-dependent.

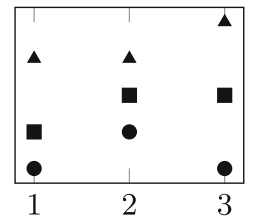

Machine

(a) Job-correlated

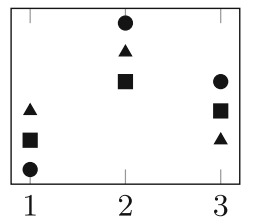

Machine

(b) Machine-correlated

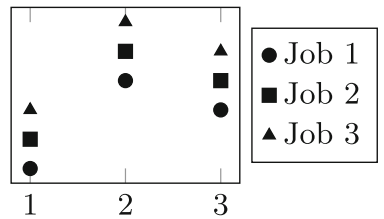

Machine

(c) Mixed-correlated

Fig. 1. Job processing time for three types of structured PFSP instances.

For each of the three correlation types, four $(J \times 20)$ sets are generated (See underlined in Table 1). For each instance size, 1100 instances are generated, with varying values for correlation: $\alpha \in\{0.0,0.1 \cdots 1.0\}$. For $\alpha=0.0$, instances reflect the way Taillard instances are generated, higher values introduce more correlation. For $\alpha=1.0$, every task in a job/machine has the same processing time.

\subsection{Comparing Results}

To compare algorithms for PFSP, the Relative Percentage Deviation (RPD) is often used. The RPD describes the relative distance to the best known upper 
Table 1. Sizes of the Taillard PFSP instances, for underlined sizes structured instances are available.

\begin{tabular}{l|l|l|l|l|l}
\hline & $J=20$ & $J=50$ & $J=100$ & $J=200$ & $J=500$ \\
\hline$M=5$ & $20 \times 5$ & $50 \times 5$ & $100 \times 5$ & & \\
\hline$M=10$ & $20 \times 10$ & $50 \times 10$ & $100 \times 10$ & $200 \times 10$ & \\
\hline$M=20$ & $\underline{20 \times 20}$ & $\underline{50 \times 20}$ & $\underline{100 \times 20}$ & $\underline{200 \times 20}$ & $500 \times 20$ \\
\hline
\end{tabular}

bound $(U B)$ of an instance and the result of the algorithm $R E S$. The RPD is calculated by

$$
R P D(R E S)=\frac{100 \cdot(R E S-U B)}{U B} .
$$

RPD values are best used when the upper bound is very close to the optimal solution. An RPD value of 0.0 then means that the optimal solution has been found. Over a set of runs, the average or median RPD is often reported (ARPD/MRPD). In our results, we also report the average over the MRPDs of multiple instances (AMRPD).

We use the Mann-Whitney-U test to check for a significant difference between two algorithms. Unless reported otherwise, we use sample sizes of 20 per instance to find MRPD values. AMRPD values are found over 10 instances with the same size. For significance tests we use a significance level of $p<0.05$.

\section{Heuristics for the PFSP}

\subsection{Constructive Heuristics}

For the TFT criterion, Liu and Reeves have introduced the LR(x) heuristic [6], which can generate up to $J$ schedules, depending on the parameter $x$. LR(x) builds a schedule from the front to the back, using the following three steps:

1. Sort all jobs according to the index function.

2. Create $x$ partial schedules with the top-x jobs scheduled first. Extend the partial schedules by iteratively adding the best job according to the re-evaluated index function.

3. Select the best schedule generated in step 2).

The index function for adding job $i$ after the last job $k$ in the partial schedule consists of two components:

1. A weighted total machine idle time, penalizing the time the machines wait between job $k$ and job $i$. Idle time on the first machines is punished more than idle time on the last machines.

2. The artificial total flow time, is the sum of the completion time of job $i$ plus the completion time of an artificial job representing the unscheduled jobs. 


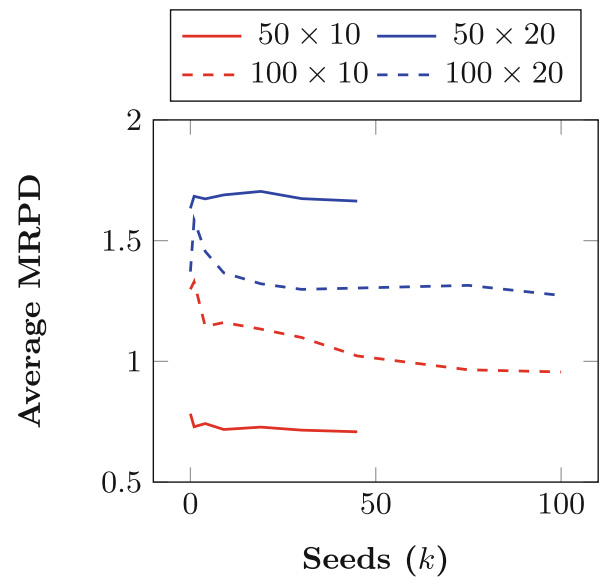

(a) Taillard instances

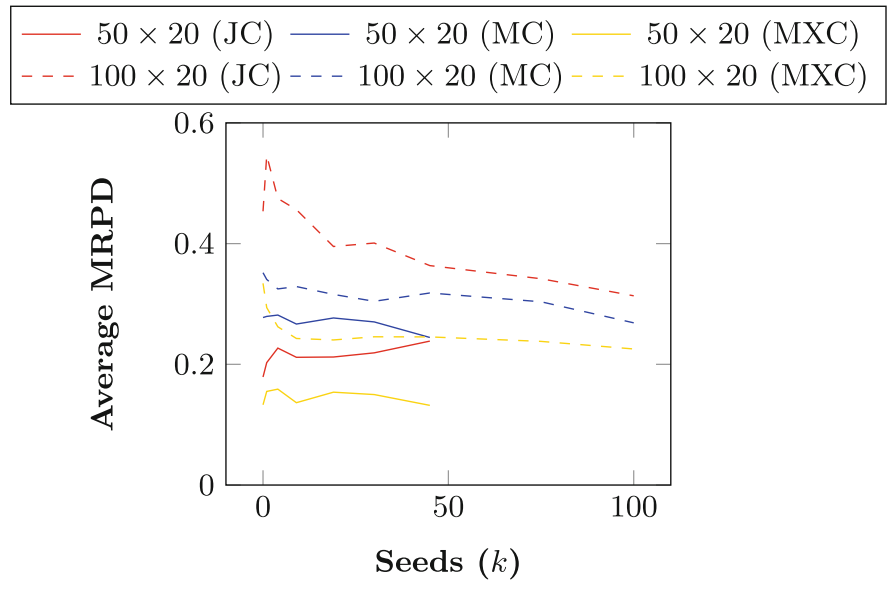

(b) Structured instances

Fig. 2. Seeding with the LR heuristics: amount of seeds vs. solution quality after $50,000,000$ fitness evaluations.

\subsection{Constructive Heuristics Seeding: Results}

For the LR heuristic we have tested the effect of seeding solutions in the initial populations of permutation GOMEA. Figure 2 shows that for most instances especially the larger ones - more seeds result in better solutions. This holds for both structured and unstructured instances. An interesting observation is the effect of single-solution seeding. Here, the dominant new solution can misguide optimal mixing, leading to worse solutions. 


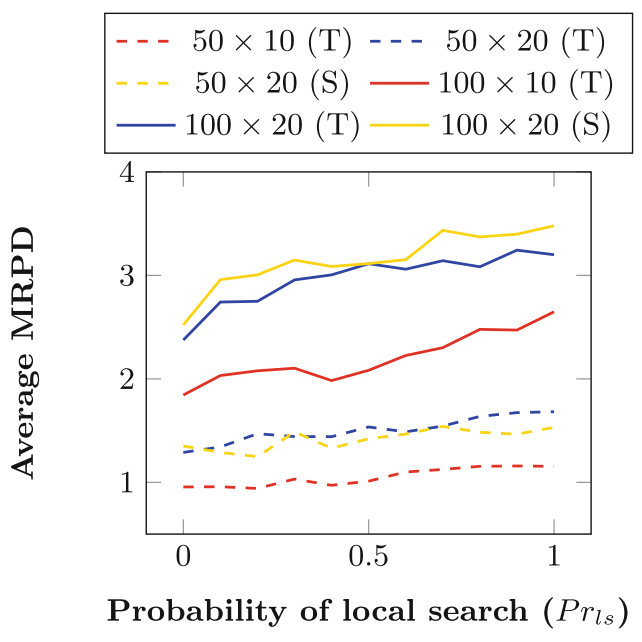

Fig. 3. Hybrid GOMEA performance with respect to the probability of local search for Taillard (T) and structured (S) instances $(\alpha=0.3)$

\subsection{Improvement Heuristics}

For the PFSP with the TFT criterion, various improvement heuristics exist. Each of these improvement heuristics are based on two fundamental permutation neighborhoods: job insertion and job swap. The swap heuristic takes two jobs and swaps them in a permutation. The insertion heuristic takes one job and puts it in another place in the permutation. Both heuristics have a neighbor-space that is quadratic in the amount of jobs and take $\mathcal{O}(J \cdot M)$ time to compute the fitness of a neighbor. In permutation GOMEA an improvement heuristic is most effectively applied when a solution has changed in the GOM phase. For permutation GOMEA solving the PFSP with the TFT criterion, the swap heuristic was shown to have the most potential, especially on instances with a few machines (for more details see [1]). Figure 3 shows for structured (mixed-correlation) and unstructured instances how permutation GOMEA performs when this improvement heuristic is applied with some probability $\operatorname{Pr}_{l s}$. Clearly, the use of the neighborhood search does not improve the effectiveness of permutation GOMEA within the given computational time budget. Apparently the extensive search already executed by the Gene-pool Optimal Mixing process does not benefit anymore from the classical swap neighborhood exploration.

\section{Permutation GOMEA vs. VNS4 Iterated Local Search}

The previous section showed that permutation GOMEA can best be enhanced by seeding the initial population with solutions constructed with the LR heuristic. Adding local search to improve each solution after the gene-pool mixing process does not result in consistent improvements on all instances, and is therefore 
Table 2. Quality of pGOMEA and VNS4 on Taillard instances.

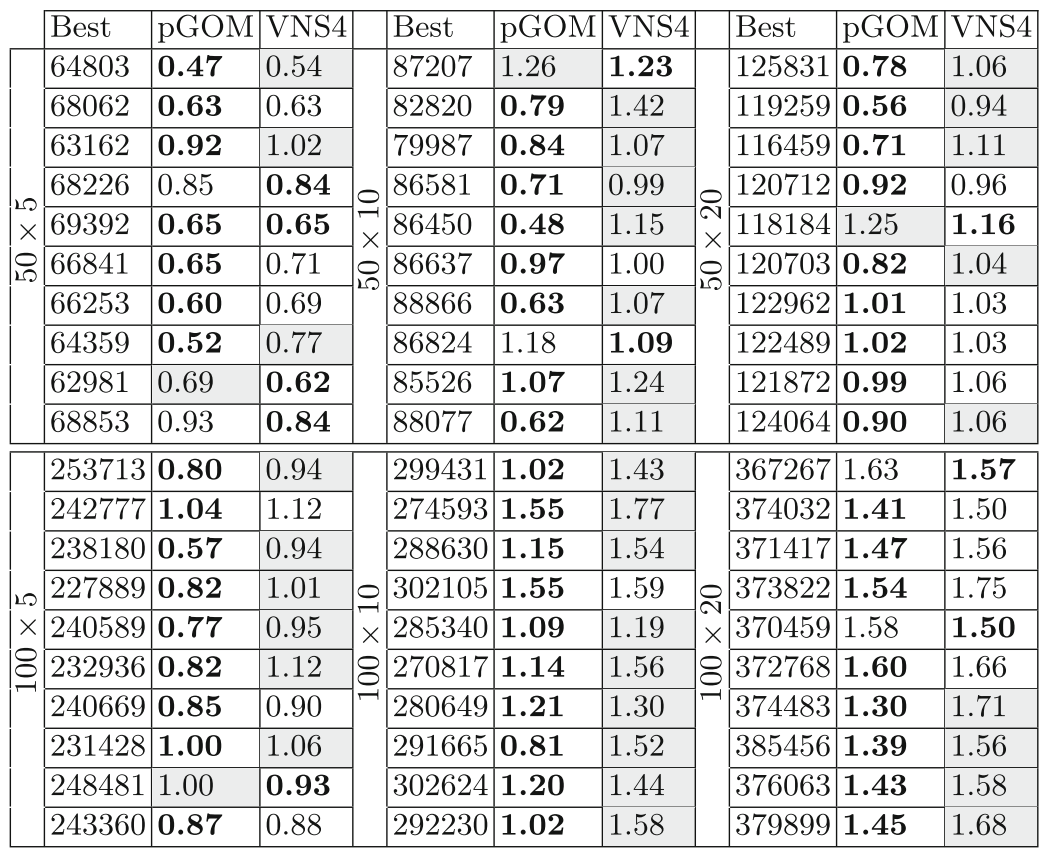

not applied in this section. To see how well permutation GOMEA performs in comparison with a well tested Iterated Local Search heuristic for the PFSP, we compare it with VNS4, a Variable Neighborhood Search algorithm which uses an optimal form of combining the insertion heuristic and swap heuristic in order to solve the PFSP with the TFT criterion [4]. VNS4 was the most successful algorithm in a study of six different ways to combine the two most used neighborhoods in the literature used for the permutation flowshop scheduling problem with total flowtime criterion, namely job interchange and job insertion. VNS4 turned out to be the most effective of the six variable neighborhood search algorithms. VNS4 was also compared to a state-of-the-art evolutionary approach which it outperformed on most of the benchmark instances.

VNS4 is started from a solution generated by the LR constructive heuristic. First, VNS4 fully explores the job interchange neighborhood until no further improvement is possible. Then, a single iteration of the job insertion neighborhood search is executed. If this iteration improves the current solution, the algorithm resumes the interchange neighborhood search. When a local optimum common to both neighborhoods has been reached within the computational time limit, VNS4 executes a random walk to escape from the region of attraction of this local optimum. The random walk consists of $k$ random job insertion moves. Iterated Local Search is sensitive to the length of the perturbation size. Experimental results show that VNS4's performance degrades when the perturbation 
Table 3. Quality of pGOMEA and VNS4 on structured instances.

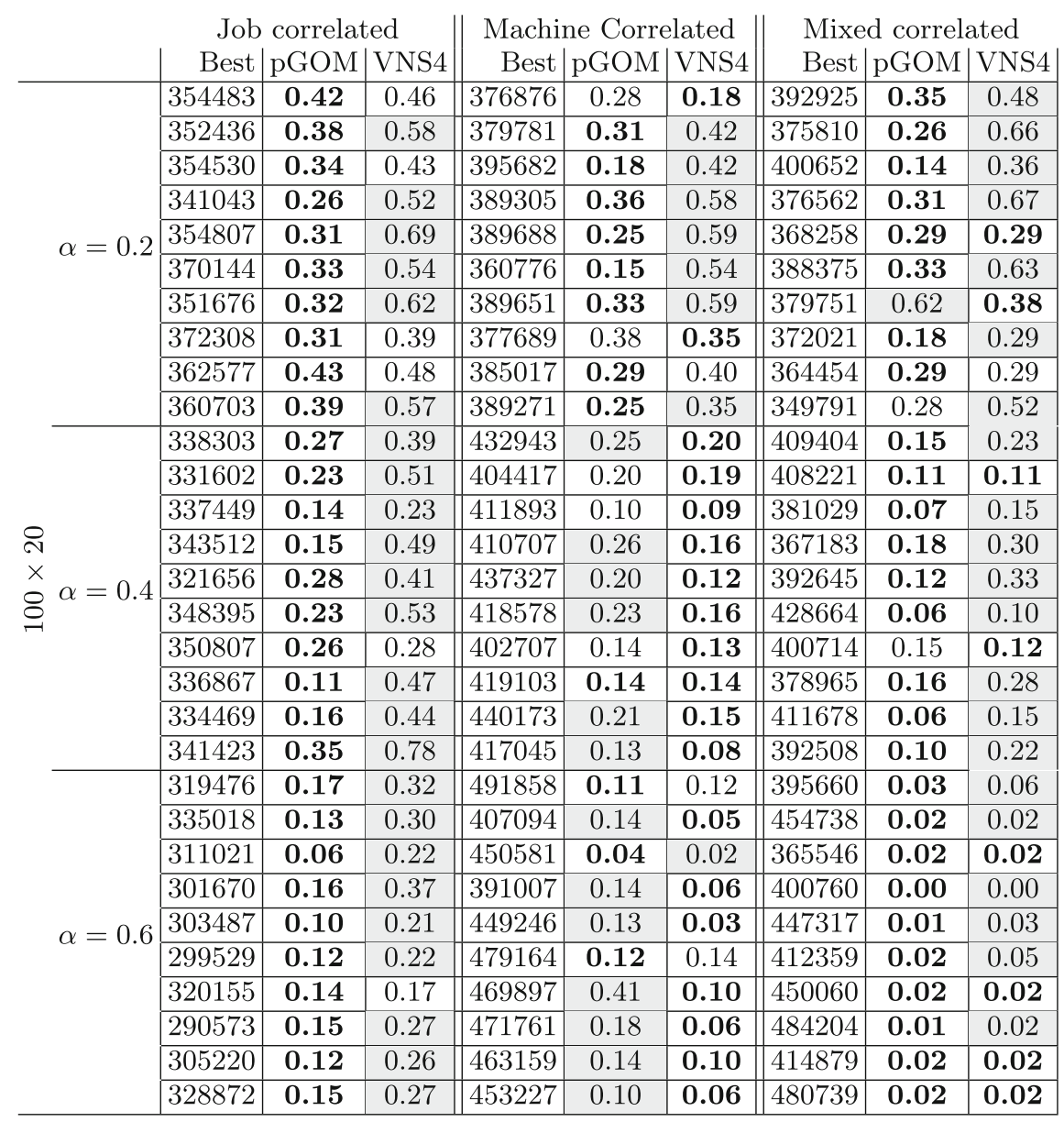

size is less than 14 or greater than 18 random job insertion moves [4]. The results with $14 \leq k \leq 18$ produce very similar results, but $k=14$ has the lowest RPD median, so this value is shown here in the Tables with experimental results.

Table 2 shows the MRPD values on Taillard problem instances for VNS4 and permutation GOMEA when both algorithms are run for $400 \cdot J \cdot M$ milliseconds. This stopping criterion is the same as used in recent works of $[5,8,11]$ which were all included in the comparison in [4].

The best solution in the Table is marked bold and if the other solution performs significantly worse, its cell is marked grey. The results show that in most cases permutation GOMEA outperforms VNS4 significantly, in a number of cases there is no statistically significant difference, and in only a few instances VNS4 outperforms permutation GOMEA. 
Secondly, we have tested permutation GOMEA and VNS4 on multiple structured instances with size $100 \times 20$. For these problems we have run the algorithms for $400 \cdot(1-\alpha) \cdot J \cdot M$ seconds, as structure makes the problems easier. Table 3 shows the results for three types of structured instances and three $\alpha$ values.

The results show for job-correlated instances that permutation GOMEA always outperforms the VNS4 algorithm. The type of structure apparently suits permutation GOMEA best, while VNS4 cannot benefit from an easier fitness landscape. The machine-correlated instances with a high amount of structure $(\alpha \geq 0.4)$ are however easier for VNS4. When machine and job correlation are mixed, the PFSP is best solved using permutation GOMEA. Permutation GOMEA finds solutions with MRPD values lower than 0.5 , showing that structured instances are easier than the standard Taillard instances.

An interesting question is why permutation GOMEA does not outperform VNS4 for the machine-correlated instances with a high amount of structure? Apparently, permutation GOMEA does not fully capture the structure in the machine-related instances. The most likely explanation is that this structure is not represented well enough in the distance measure used to build the linkage tree. Further research into the relation between the structure in specific problem instances and the type of structure searched for by GOMEA using different distance measures is needed to answer this question.

\section{Conclusions}

Previous work has shown how the Gene-pool Optimal Mixing Evolutionary Algorithm can be applied to permutation problems like the PFSP by representing solutions with the random-key encoding. Each generation GOMEA builds a linkage tree in order to capture structure in the set of solutions. This linkage tree can also be looked upon as an adaptive neighborhood learned by GOMEA to explore new solutions. In this paper we have investigated how the use of constructive heuristics and neighborhood search might improve on the Black-Box approach of permutation GOMEA. Results showed that adding neighborhood search does not consistently improve the performance. However, seeding the initial population of GOMEA by solutions generated by the constructive LR heuristic was shown to be an effective technique. We have experimentally compared permutation GOMEA - seeded with the constructive heuristic LR - with the highly successful VNS4 algorithm for unstructured and structured Permutation Flowshop Scheduling problems. VNS4 is an Iterated Local Search algorithm using a variable neighborhood that combines the job insertion neighborhood with the job swap neighborhood.

For the unstructured Taillard instances, GOMEA almost always outperforms VNS4. Also for the job correlated structured instances and for the mixed job/machine correlated instances GOMEA outperforms VNS4. Only for machine correlated structured instances with a high amount of structure $(\alpha \geq 0.4)$, VNS4 outperforms permutation GOMEA.

As a general conclusion, this paper has shown that the use of a multi-solution constructive heuristic to seed the initial population of permutation GOMEA 
leads to an effective model-based evolutionary algorithm. It has also been shown that adding neighborhood search algorithms does not always result in more efficient results given a fixed computational time budget.

\section{References}

1. Aalvanger, G.: Incorporating domain knowledge in permutation gene-pool optimal mixing evolutionary algorithms. Master's thesis. Utrecht University, The Netherlands (2017). https://dspace.library.uu.nl/handle/1874/353005

2. Bosman, P.A., Luong, N.H., Thierens, D.: Expanding from discrete Cartesian to permutation gene-pool optimal mixing evolutionary algorithms. In: Proceedings of the Genetic and Evolutionary Computation Conference, pp. 637-644. ACM (2016)

3. Ceberio, J., Irurozki, E., Mendiburu, A., Lozano, J.A.: Extending distance-based ranking models in estimation of distribution algorithms. In: 2014 IEEE Congress on Evolutionary Computation, CEC, pp. 2459-2466, July 2014

4. Costa, W.E., Goldbarg, M.C., Goldbarg, E.G.: New VNS heuristic for total flowtime flowshop scheduling problem. Expert Syst. Appl. 39(9), 8149-8161 (2012)

5. Jarboui, B., Eddaly, M., Siarry, P.: An estimation of distribution algorithm for minimizing the total flowtime in permutation flowshop scheduling problems. Comput. Oper. Res. 36, 2638-2646 (2009)

6. Liu, J., Reeves, C.R.: Constructive and composite heuristic solutions to the $\mathrm{p} \| \sum C_{i}$ scheduling problem. EJOR 132(2), 439-452 (2001)

7. Taillard, E.: Benchmarks for basic scheduling problems. Eur. J. Oper. Res. 64(2), 278-285 (1993)

8. Tasgetiren, M.F., Pan, Q.-K., Suganthan, P.N., Chen, A.H.-L.: A discrete artificial bee colony algorithm for the permutation flow shop scheduling problem with total flowtime criterion. In: Proceedings of the IEEE World Congress on Computational Intelligence, WCCI-2010, pp. 137-144. IEEE (2010)

9. Thierens, D., Bosman, P.A.: Optimal mixing evolutionary algorithms. In: Proceedings of the Genetic and Evolutionary Computation Conference, pp. 617-624 (2011)

10. Watson, J.-P., Barbulescu, L., Whitley, L.D., Howe, A.E.: Contrasting structured and random permutation flow-shop scheduling problems. INFORMS J. Comput. 14(2), 98-123 (2002)

11. Xu, X., Xu, Z., Gu, X.: An asynchronous genetic local search algorithm for the permutation flowshop scheduling problem with total flowtime minimization. Expert Syst. Appl. 38, 7970-7979 (2011) 